LMU-12/94

\title{
Exact Cancellation of Quadratic Divergences in Top Condensation Models
}

\author{
Andreas Blumhofer \\ Sektion Physik \\ Ludwig-Maximilians-Universität München \\ Theresienstr.37, D-80333 München
}

\begin{abstract}
We discuss the hierarchy problem and the corresponding quadratic divergences in the top mode Standard Model. Quadratic divergences appear at each order $1 / N_{c}$ since fermionic and bosonic contributions are of different order $1 / N_{c}$. It is shown that the full dynamical system to all orders in $1 / N_{c}$ admits a solution, where the sum of all quadratic divergent contributions disappears.
\end{abstract}

${ }^{*}$ Email: ab at hep.physik.uni-muenchen.de 


\section{Introduction}

In spite of the successful description of the strong and electroweak phenomena by the Standard Model, the prediction of the particle masses is still a big challenge. Although the Higgs sector of the Standard Model explains the electroweak symmetry breaking and how particles acquire a mass, it only seems to be an effective description due to the many parameters. We are still far away from a theory being able to predict all known particle masses and mixing angles in the Kobayashi-Maskawa-matrix. However in a first step such a theory should at least predict the $\mathrm{W}$ - and $\mathrm{Z}$-masses. If there is a great desert, then it should also explain, why the Grand Unification scale is so many orders of magnitude beyond the weak scale. In the Standard Model this leads to the so-called hierarchy problem, which manifests itself in quadratic divergences of the Higgs tadpoles and self-energies. Of course, if the Standard Model is the final theory, then quadratic divergences are eliminated by renormalization. But if the divergences originate from a dynamical interaction generating the particle masses, the cutoff $\Lambda$ will be the scale of that interaction. In that case the quadratic divergences give huge contributions for the particle masses of the Standard Model which make their low values unstable.

At the one-loop-level (fig. 国) they have the form

$$
\left[4\left(m_{t}^{2}+m_{b}^{2}+\frac{1}{3} m_{\tau}^{2}+\ldots\right)-M_{H}^{2}-M_{Z}^{2}-2 M_{W}^{2}\right] \Lambda^{2},
$$

where all fermions and bosons contribute according to their degrees of freedom. In trying
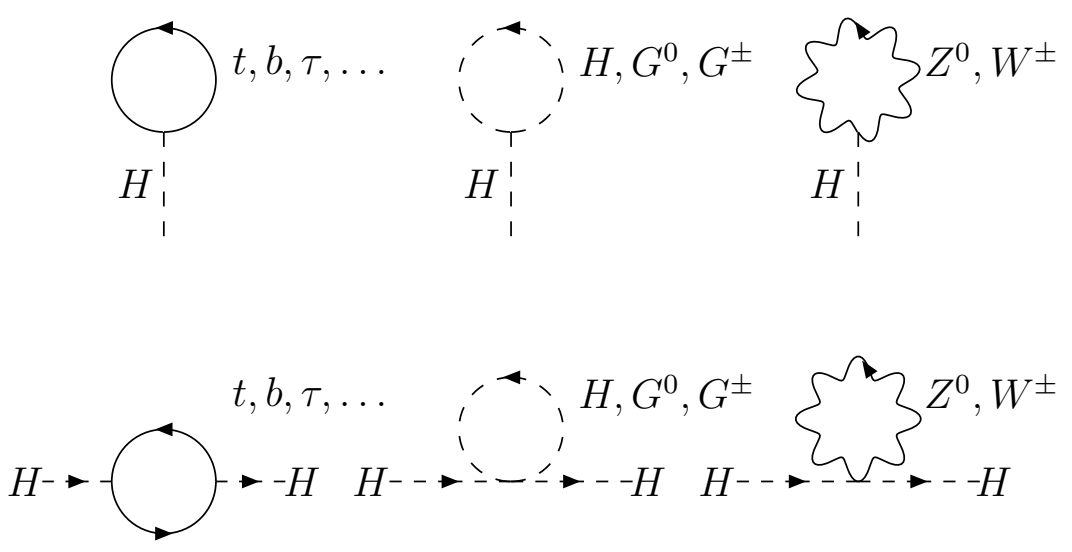

Figure 1: One-loop quadratic divergent Standard Model graphs.

to solve the hierarchy problem one can demand the cancellation of quadratic divergences 
in lowest order, namely the vanishing of (1), called Veltman condition, first mentioned by R. Decker and J. Pestieau [1]. Unfortunately that condition requires an unnatural finetuning of independent masses, which is not enforced by symmetries. Moreover this relation is within the Standard Model completely regularization scheme dependent, i. e. if we use different cutoffs for different particle loops we get different conditions. Hence in the pure Standard Model the Veltman condition has no meaning. This is different, if the Standard Model arises as an effective low energy Lagrangian from some new dynamics at a high scale. We will see that formfactors of composite particles and masses of the underlying theory act then like physical cutoffs leading to an effective Veltman condition.

The regularization scheme dependence drops out, if a corresponding symmetry is present. A big progress in this direction was made with supersymmetric field theories凹, which are free of quadratic divergences because of the same bosonic and fermionic degrees of freedom of the superpartners. So far supersymmetry is the only theory which avoids that hierarchy problem. Nevertheless the many new parameters in supersymmetric theories make the particle mass problem worse since the Higgs mechanism is not replaced by an underlying theory but is legitimated as fundamental. Thus many authors tried to solve the hierarchy problem for the Standard Model and for non-supersymmetric extensions [3, 4, 5]. They dealt with the Veltman condition and further additional assumptions. The two loop cancellation of quadratic divergences in the Standard Model [4] leads however to a further condition and higher orders surely make the situation even worse. Some authors emphasized that in renormalizable scalar theories and sigma models the higher order quadratic divergences are related to the lowest order ones [5]. But this theorem failed at the four-loop-level.

It seems that a cancellation does not happen by accident. In the spirit of supersymmetry one needs an additional relation between fermions and bosons leading to cancellations in a natural way. Nambu first argued that in a composite Higgs model the masses of the expression (I) are not independent and could automatically be arranged in such a way that the quadratic divergences cancel [6]. In the proposed model without new fermions the Higgs mainly consists of a top-antitop-pair tightly bounded by new interactions. One expects therefore $M_{H} \approx 2 m_{t}$, which roughly coincides with the vanishing of (11). Nambu introduced the term "Quasi-Supersymmetry" because the Higgs and the top play the role of "Superpartners" in this model. But in such theories the Higgs is a dynamical object and all masses result from a complicated system of Schwinger-Dyson-equations. Since dynamics and not symmetry sets the mass relations it is however hard to believe that they

\footnotetext{
${ }^{1}$ for a review see [2]
} 
fulfill the Veltman condition exactly.

In a series of papers [7] the top condensation idea was worked out by several authors. Nevertheless the meaning of the Veltman condition for top condensation was so far unclear. In a recent paper [8] it was shown that a cancellation of quadratic divergences appears in such top condensation models in lowest order of a combined color-flavor-expansion. The reason is that like in supersymmetry the fermion and boson couplings, i. e. in top condensation the top Yukawa-coupling and the Higgs self-coupling, are related since the Higgs is a $\bar{t} t$-boundstate. In addition the number of fermion and boson degrees of freedom are not equal in contrast to supersymmetry but matched in such a way that the quadratic divergences cancel. This cancellation does however not require a mass relation between the top and the Higgs mass and the Veltman condition, which was the motivation for

this scenario, disappears. Nevertheless so far no symmetry was found as the origin of the cancellation and the behaviour of the higher order $1 / N_{c}-$ corrections was therefore unclear.

Now in this paper I want to emphasize that the above mechanism seems to work, if we include all orders $1 / N_{c}$, so that the quadratic divergences cancel exactly. In section II top condensation and how the hierarchy problem appears in that model is recapitulated. Section III explains the mechanism leading to the cancellation of quadratic divergences in top condensation for a certain approximation independent of the values of top and Higgs mass. That cancellation has another meaning than the usual Veltman condition. Contrasts and relations are shown in section IV. The rather technical section V includes a complete proof of the cancellation to all orders, which follows from a solution of the full system of Schwinger-Dyson-equations. Section VI emphasizes that in a general context this solution does not appear for other gauge groups and prefers the Standard Model as the only possible choice of group parameters leading to a cancellation of quadratic divergences. Finally section VII handles with the vector-boson contributions and their influence on the cancellation condition.

\section{The hierarchy problem in top condensation}

Let us first consider the minimal top condensation model in lowest order $1 / N_{c}$. It consists of the Standard Model without Higgs sector but with the additional four-fermion-interaction

$$
\mathcal{L}_{I}=\frac{G}{N_{c}}\left(\overline{\psi_{L}} t_{R}\right)\left(\overline{t_{R}} \psi_{L}\right) ; \quad \psi_{L}=\left(\begin{array}{c}
t_{L} \\
b_{L}
\end{array}\right)
$$


where color is summed in the brackets. This model was studied by W. A. Bardeen, C. T. Hill and M. Lindner (BHL) [7]. We imagine that this four-fermion-interaction is generated by new heavy bosons at a high scale $M$, far above the TeV-region. Therefore higher dimensional operators are suppressed and the special structure of this new interaction drops out [9].

After a reasonable choice of the auxiliary fields $H, G^{0}$ and $G^{ \pm}$one finds the usual Standard Model Higgs sector, where the kinetic terms for the Higgs field components are absent:

$$
\begin{aligned}
& \mathcal{L}_{I}=-\frac{1}{2} \frac{g_{t}^{2} N_{c}}{G}\left(H H+G^{0} G^{0}+2 G^{+} G^{-}\right) \\
& -\frac{g_{t}}{\sqrt{2}} H \bar{t} t+i \frac{g_{t}}{\sqrt{2}} G^{0} \bar{t} \gamma^{5} t+g_{t}\left(G^{+} \bar{t} L b+G^{-} \bar{b} R t\right) \text {. }
\end{aligned}
$$

At tree level the Higgs and the Goldstone bosons do not propagate. Their "static" propagator has the form:

$$
i \frac{G}{g_{t}^{2} N_{c}}
$$

Two Yukawa couplings connected by that static propagator is nothing else than a fourfermion-vertex. The Lagrangian (3) is therefore completely equivalent to the four-fermioninteraction (2).

The top mass is generated by the gap equation which reads in lowest order $1 / N_{c}$ (see fig. 2):

$$
1=\frac{2 G}{(4 \pi)^{2}}\left(M^{2}-m_{t}^{2} \ln \frac{M^{2}}{m_{t}^{2}}\right) .
$$

The bright circle is the self-energy and the dark circle the propagator, where the self-

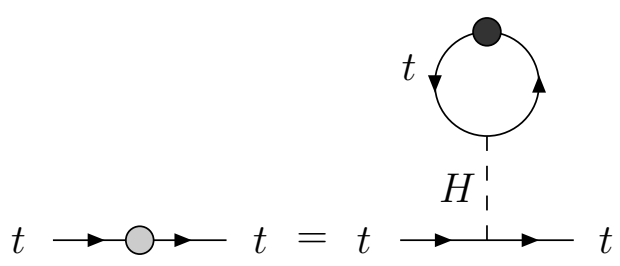

Figure 2: Gap equation in lowest order $1 / N_{c}$.

energies are summed up. 


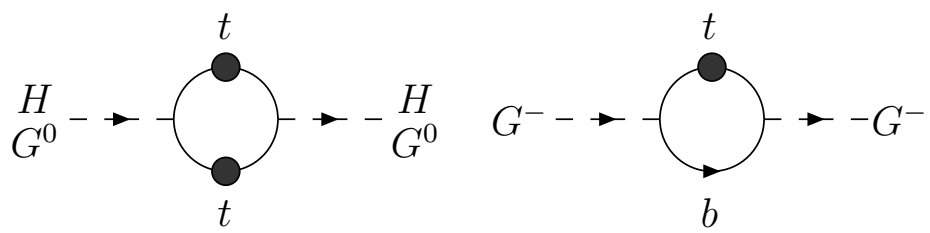

Figure 3: Boson self-energies in lowest order $1 / N_{c}$.

Including the resummation of the lowest order $1 / N_{c}$ boson graphs (fig. 3i) the auxiliary fields become propagating boundstates. The propagators are:

$$
\begin{aligned}
D_{H} & =\zeta^{-1} \frac{i}{p^{2}-4 m_{t}^{2}} \\
D_{G^{0}} & =\zeta^{-1} \frac{i}{p^{2}} \\
D_{G^{ \pm}} & =\zeta^{-1} \frac{i}{p^{2}}
\end{aligned}
$$

where

$$
\zeta=\frac{N_{c} g_{t}^{2}}{(4 \pi)^{2}} \ln \frac{M^{2}}{p^{2}}
$$

up to finite terms. From (6) we get the well known relation $M_{H}=2 m_{t}$.

An important test for a mechanism explaining the electroweak symmetry breaking is the right prediction of the $\mathrm{W}$ - and $\mathrm{Z}$-mass. The main contributions are the fermion-loop diagrams in fig. 因. In the minimal model we find:

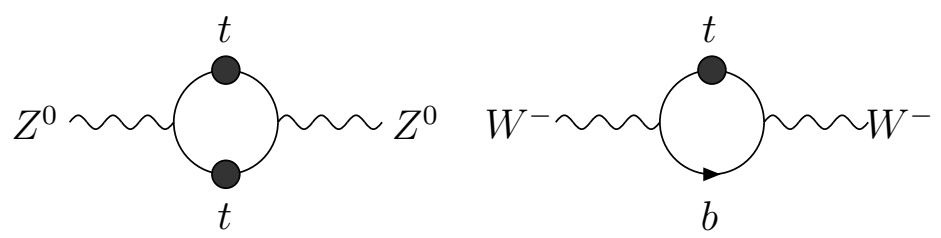

Figure 4: Vector-boson self-energies in lowest order $1 / N_{c}$.

$$
\begin{aligned}
M_{W}^{2} & =\frac{N_{c} g_{2}^{2}}{2(4 \pi)^{2}} m_{t}^{2} \ln \frac{M^{2}}{m_{t}^{2}} \\
M_{Z}^{2} & =\frac{N_{c}\left(g_{1}^{2}+g_{2}^{2}\right)}{2(4 \pi)^{2}} m_{t}^{2} \ln \frac{M^{2}}{m_{t}^{2}}
\end{aligned}
$$


which predicts the $M_{Z} / M_{W}$ ratio of the Standard Model and leads to the condition:

$$
1=\frac{N_{c} g_{t}^{2}}{(4 \pi)^{2}} \ln \frac{M^{2}}{m_{t}^{2}}
$$

For $g_{t} \approx 1$ one gets $M \approx 5 \cdot 10^{13} \mathrm{GeV}$. For such a high cutoff the gap equation (5) runs into a fine-tuning problem because $m_{t} \approx M$ for almost the whole range of the G-parameter. Therefore more realistic top condensation models [10] dealt with a TeV-cutoff, where the predicted ratio for $M_{W} / m_{t}$ is to low. These models include a strong interaction with new heavy gauge bosons with mass $M_{X}$, which reproduces the four-fermion-interaction (2) in the low energy limit. The corresponding gap equation (fig. 5) provides a $p^{2} / M_{X}^{2}$ growth of

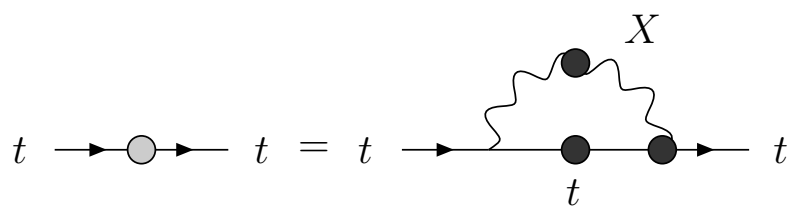

Figure 5: Gap equation with massive vector-bosons.

the dynamical top mass below $M_{X}$. This is closely related to the appearence of quadratic divergences in the low energy regime where only a four-fermion-interaction remains. For large $M_{X}$ this strong running provides the hierarchy problem, i. e. the gap equation can not stabilize a low top mass apart from a very tiny window of couplings according to eq. (5). The situation drastically changes, if the low energy theory is free of quadratic divergences which enables the top mass to run logarithmically towards small $p^{2}$ after shortly falling down at the scale $M_{X}$. This coincides with the usual renormalization group equation of the Standard Model where a large enough top mass is running very smoothly up to the Landau pole, where it blows up very fast.

In the following we will show that the low energy theory is indeed free of quadratic divergences, so that the scale $M_{X}$ can be chosen very high to get a reasonable $\mathrm{W}$-mass value without running into a fine-tuning problem. 


\section{Cancellation of quadratic divergences in a prelim- inary approximation}

So far the gap equation (5) is quadratic divergent. After including of higher order diagrams the quadratic divergent contributions need a reshuffling of Feynman graphs to be obvious. We will further show in the following the cancellation of these contributions in a preliminary approximation.

From eq. (6) we have seen that the lowest order $1 / N_{c}$ Higgs propagator yields the relation $M_{H}=2 m_{t}$. We therefore expect that a cancellation of quadratic divergences appears, at least partly, due to the Veltman condition. Unfortunately this is not the case, since the lowest order $1 / N_{c}$ gap equation (fig. 2) contains only a fermion-loop tadpole. All the other tadpoles of fig. 1 with the opposite sign are of higher order $1 / N_{c}$ or $g_{1 / 2}^{2}$. They drop out although Higgs and Goldstone boson diagrams are numerically of the same order as the fermion tadpole. Hence in lowest order $1 / N_{c}$ it is impossible to see any cancellation. From that one has to go beyond the leading order. We neglect at the moment vector-boson contributions which will be considered later.

The exact Schwinger-Dyson-equations for the top and Higgs self-energies are shown in fig. 6. The full vertices are defined by their skeleton expansion. The Higgs line in the

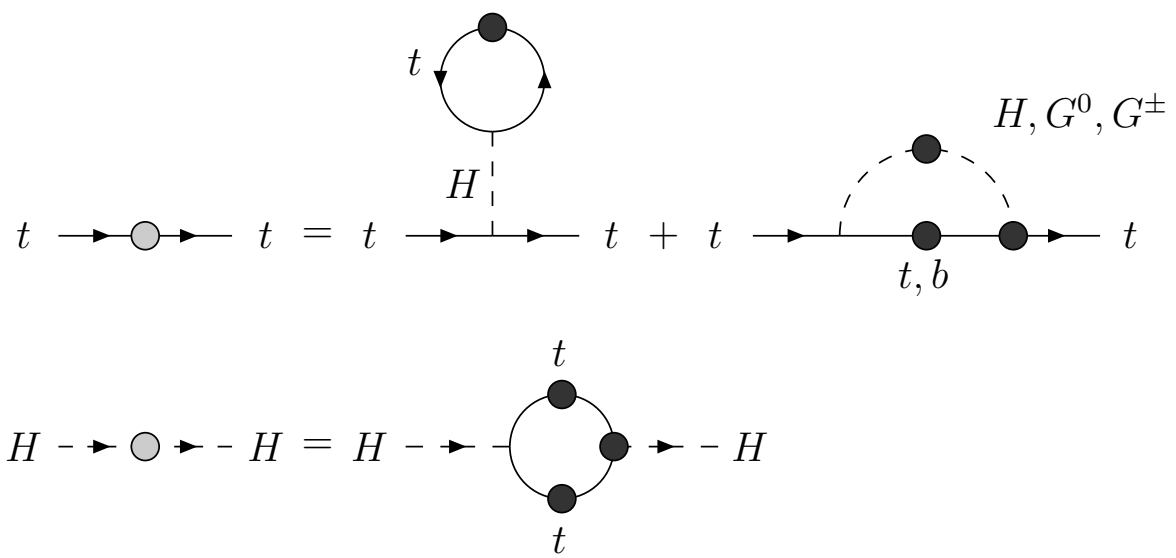

Figure 6: Exact Schwinger-Dyson-equations.

tadpole must be the non-propagating auxiliary field with bare vertices to avoid double counting]. Tadpoles with boson-loops as in fig. 1 1 again do not appear in the top self-

\footnotetext{
${ }^{2}$ E.g. if one inserts a fermion-loop in the Higgs propagator, the upper part of the diagram is a top self-energy correction for the tadpole with the new top-loop. This graph is already considered in fig. 6 .
} 
energy. They would provide quadratic divergences with opposite sign to cancel the fermion contribution. These graphs are implicitly involved by reinsertion of the last diagram into the tadpole. To make them obvious one has to extract the tadpole contribution as in the recent paper [8]. We define the tadpole by the box (see fig. 7) and replace all fermion self-energy circles with boxes according to the first line in fig. 6, i.e. the last diagram of the first line in fig. 6 is not included in the box and must be explicitly inserted in the fermion-loop of the tadpole several times, where the full vertex is replaced by the skeleton expansion.

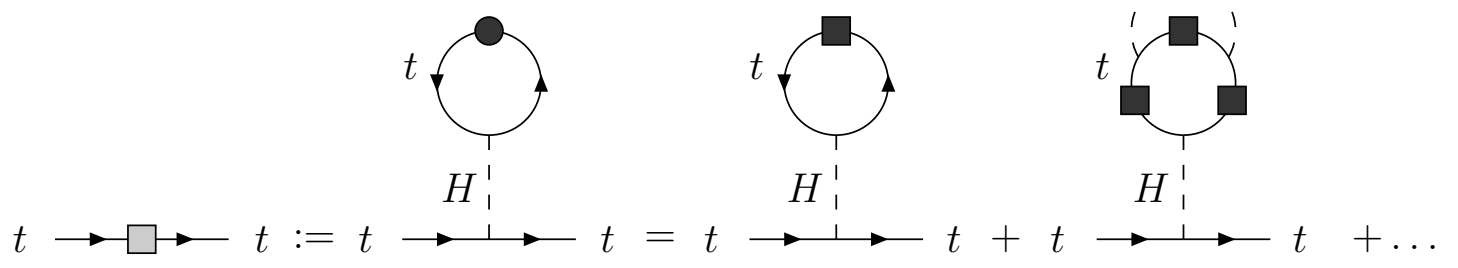

Figure 7: Redefined top self-energy.

Since the tadpole just provides a mass correction for the top quark, this also insures that the top propagator with the box does not receive wave function corrections, which is essential for further discussions. Using this new definition the boson self-energies can also be written in terms of the box propagator (see fig. 8).

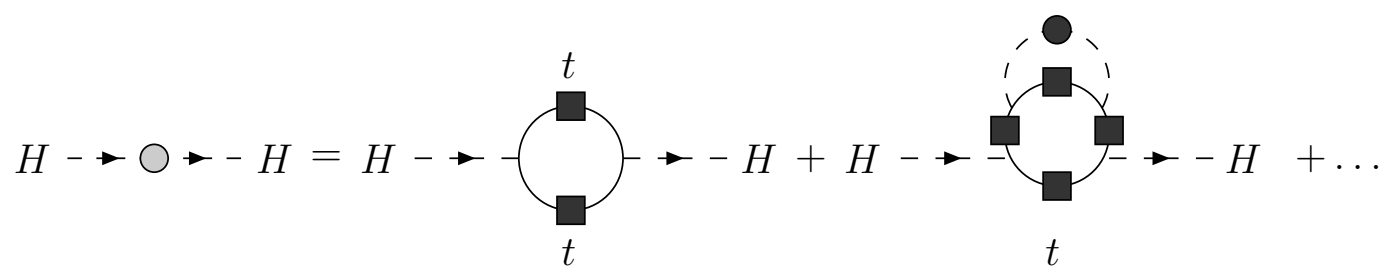

Figure 8: Exact Higgs boson self-energy.

In fig. 7 and fig. 8 we get diagrams which appear if we replace the three- and four-bosonvertices of the Standard Model in fig. 11 by fermion-loops. Such fermion-loop induced vertices can easily be calculated up to finite terms. One finds a simple relation between the fermion-loop induced and the corresponding Standard Model tree vertices. One must only multiply the Standard Model vertex $\Gamma_{\mathrm{SM}}$ with the following factor to get the top 
condensation effective vertex $\Gamma$ :

$$
\Gamma=\Gamma_{\mathrm{SM}} \zeta\left(\frac{2 m_{t}}{M_{H}}\right)^{2}+\text { finite terms. }
$$

The $\zeta$ is defined in eq. (9) and depends on the largest momentum in the diagram. This factor is universal for all Higgs and Goldstone boson vertices. All higher $\mathrm{n}-$ point functions, which do not exist in the Standard Model, are finite in top condensation and therefore negligible for the discussion of the leading divergences.

In the following we discuss the quadratic divergences of the Higgs self-energy. For simplicity we consider at the moment only self-energies with at most one inner boson line. To avoid an overkill of dots, boxes, etc. we do not write the box for the fermion propagator and the dot for the boson propagator explicitly from now on.

In our preliminary approximation we consider the remaining graphs in fig. 9, where the four-boson-vertex is simply replaced by a fermion loop?. To calculate the quadratic diver-

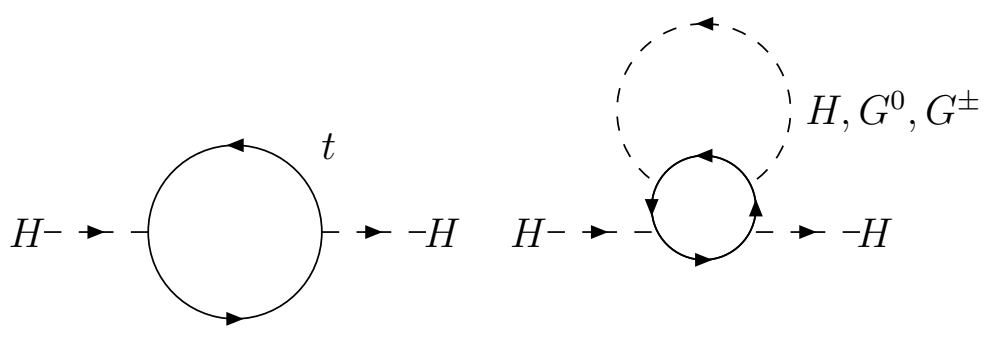

Figure 9: Quadratic divergent graphs in a preliminary approximation.

gences one can use the Standard Model formulae for both graphs, where in top condensation two additional factors enter the formula for the second graph. The propagator gets an $\zeta^{-1}$ due to eq. (6), the effective vertex a factor $\zeta\left(2 m_{t} / M_{H}\right)^{2}$ from eq. (13) $)^{\text {t }}$. We end up with the top condensation expression:

$$
\left[4 m_{t}^{2}-M_{H}^{2} \cdot \zeta^{-1} \cdot \zeta\left(\frac{2 m_{t}}{M_{H}}\right)^{2}\right] M^{2}
$$

which surprisingly vanishes independent of the values of the top and the Higgs mass. The reason for the cancellation is that the effective four-boson-vertex and the top Yukawa-

\footnotetext{
${ }^{3}$ In the second diagram all topologies of the fermion-loop generated by the order of passing through the diagram are involved although only one is shown in fig. 9 .

${ }^{4}$ Both $\zeta$ 's depend on the same loop momentum and cancel therefore.
} 
coupling are related in a certain way and that the degrees of freedom are matched. Nevertheless the cancellation happens for a mixed order $1 / N_{c}$. The first graph is of the order $N_{c}$ the second of the order 1 1 . The expression (14) has the following general form:

$$
\left[\frac{4 N_{c}}{3} m_{t}^{2}-M_{H}^{2} \cdot \zeta^{-1} \cdot \zeta\left(\frac{2 m_{t}}{M_{H}}\right)^{2}\right] M^{2},
$$

which only vanishes for $N_{c}=3$. The cancellation obviously depends on the group structure. On the contrary the $M_{H}=2 m_{t}$ relation from eq. (6) does not depend on $N_{c}$. Thus the similarity of both equations is non-trivial.

Although the graphs in fig. 9 are of different order $1 / N_{c}$, both diagrams are of the same order of magnitude. Instead of the color factor $N_{c}$ the second graph is enhanced by the number of the Higgs field components. Hence, in a recent paper [8], I have used a combined expansion in the color and flavor degrees, where both graphs are of the same order. In this paper we do not need any special expansion since we will calculate the quadratic divergences to all orders $1 / N_{c}$.

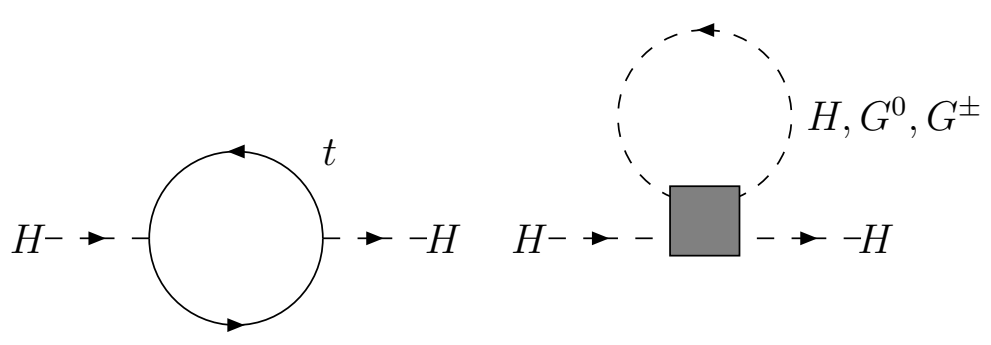

Figure 10: All order quadratic divergent graphs.

We get the full set of the Higgs self-energy diagrams in fig. 8 by replacing the fermion-loop in fig. 9 by the full four-boson-vertex, the box in fig. 10. It is clear that the quadratic divergences cancel to all orders if the full vertex is just the fermion-loop induced vertex. This happens indeed, which we will see in section $\mathrm{V}$.

\section{Low energy limit versus effective theory}

Before we go into technical details of the full analysis I like to clarify the meaning of the cutoff $M$ for the cancellation. Since the Veltman condition is regularization scheme

\footnotetext{
${ }^{5}$ Each fermion-loop provides a factor $N_{c}$ and each boson line a factor $1 / N_{c}$.
} 
dependent one has to worry about the use of equal cutoffs in eq. (14). In contrast to the Standard Model we must realize that the four-boson-vertex is dynamically generated and that both graphs of fig. 10 come from the same Higgs self-energy diagram in fig. 6. Now the above cancellation means that the vertex and the fermion propagator in the boson self-energy of fig. 6 are arranged in such a way that the last fermion-loop integration is not quadratic divergent.

One could argue that the vertex and the propagators contain different formfactors and therefore different cutoffs. As shown in the preliminary approximation and in the full analysis below the $\zeta$ 's and hence the formfactors drop out, at least in the BHL-model. The reason is that the BHL-model is dominated by the single scale $M$. A special top condensation model needs a more precise analysis to proof the formfactor independence.

On the contrary top condensation generates the Standard Model as an effective theory. How can the absence of quadratic divergences be translated into the picture of the effective theory? Does the above cancellation mean that the Veltman condition in the Standard Model is fulfilled and that $M_{H} \approx 2 m_{t}$ ? The effective theory contains a fundamental fourboson-vertex. Hence one has to use different cutoffs for fermion and boson-loops since the Veltman condition is regularization scheme dependent. We get in lowest order:

$$
4 m_{t}^{2} \Lambda_{t}^{2}-M_{H}^{2} \Lambda_{H}^{2} \approx 0
$$

or

$$
\frac{\Lambda_{t}}{\Lambda_{H}} \approx \frac{M_{H}}{2 m_{t}}
$$

The knowledge of the cancellation of quadratic divergences in the low energy limit does not help to fix the masses in the effective theory and only predicts the ratios of the different cutoffs. This is precisely the difference between the cancellation of quadratic divergences in a theory, where the new interaction is specified, and the Standard Model, where it is not. That difference is implied by the cutoff notation $M$ and $\Lambda$.

\section{Cancellation to all orders}

The aim of this section is the step by step development of the quadratic divergences of the full set of diagrams in fig. 10, i. e. we have to determine the full four-boson-vertex. It

is the solution of a complicated system of Schwinger-Dyson-equations. For the quadratic 
divergences it is sufficient to consider a subsystem of diagrams, which will be established and solved in the following.

Before we discuss the full four-boson-vertex we have to consider higher order contributions to the kinetic part of the boson self-energy. In other words: does the $\zeta$ get higher order corrections? We have to look for $p^{2} \ln \frac{M^{2}}{p^{2}}$-terms in the boson self-energy of fig.10. As we will see in this section the full four-boson-vertex is proportional to $\ln \frac{M^{2}}{p^{2}}$, so that the second graph cannot provide $p^{2} \ln \frac{M^{2}}{p^{2}}$-terms. Thus the lowest order kinetic term of the first graph and therefore $\zeta$ does not get further corrections.

Now the full four-boson-vertex contains graphs which consist of boson propagators and top-loop induced boson-vertices. It seems to be a Sisyphus work to find all four-bosondiagrams which contribute to the leading divergence. In the Standard Model this would be surely not possible. In top condensation, however, the boson propagators, which contain a factor $\zeta^{-1}$, partly remove logarithmic divergences so that $\left(\ln \frac{M^{2}}{p^{2}}\right)^{n}$-terms with $n \geq 2$ never appear. As a consequence we only have to extract simple logarithmic divergent diagrams.

The best way to see which graphs are involved is to use Euler's polyhedron theorem:

$$
\text { corners }+ \text { plains }=\text { edges }+2
$$

which give a relation for the number of corners, plains and edges of a polyhedron. In our diagram the corners are the boson vertices, the edges are the boson propagators and the plains are the loops +1 to close the polyhedron. One finds

$$
\text { vertices }+ \text { loops }- \text { propagators }=1 \text {. }
$$

This is a power equation for $\ln \frac{M^{2}}{p^{2}}$, because the vertices contain a $\ln \frac{M^{2}}{p^{2}}$, the propagators a $\left(\ln \frac{M^{2}}{p^{2}}\right)^{-1}$ and the whole diagram must be proportional to $\ln \frac{M^{2}}{p^{2}}$. As a consequence each boson-loop must be logarithmic divergent and may only consist of two boson propagators. Hence propagators $=2 \cdot$ loops and together with eq. $(\sqrt{19})$ we get $4 \cdot$ vertices $-4=2 \cdot$ propagators which is only true, if there are only four-boson-vertices. A possible graph is shown in fig. 11. The diagram does not only contain boson-loops with two boson lines. But if one calculates all such loops with two boson lines, contracts them to a point and repeats this procedure enough times, one can contract the whole graph to a point. Therefore two boson lines belong to each loop. To construct all possible diagrams one has to go just the other way round. We start with $\mathrm{t}-$ and $\mathrm{s}-$ channel bubble chains and replace the vertices again by bubble sums and so on. We end up with the self-consistent system of SchwingerDyson-equations in fig. 12, where we sum over $k, l, \ldots$ The indices run from 1 to 4 , which denote the four Higgs field components $H, G^{0}, G^{1}, G^{2}$. 


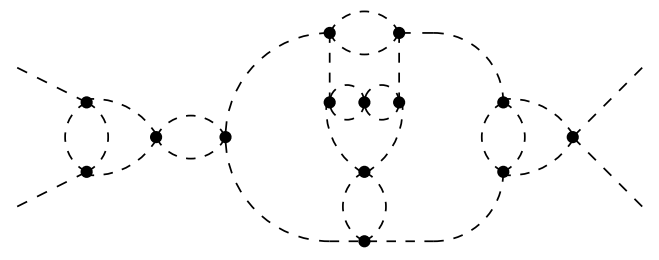

Figure 11: Possible contribution to the full vertex.

There are three types of vertices denoted by a dot, a bright circle and a dark circle. The dot is the usual fermion-loop. One therefore finds:

$$
\Gamma_{d o t}^{i j}=\Gamma_{S M}^{i j} \cdot \zeta\left(\frac{2 m_{t}}{M_{H}}\right)^{2}=\left(-i 2 g_{t}^{2} \zeta\right) \cdot A_{i j} \quad \text { where } A=\left(\begin{array}{cccc}
3 & 1 & 1 & 1 \\
1 & 3 & 1 & 1 \\
1 & 1 & 3 & 1 \\
1 & 1 & 1 & 3
\end{array}\right)
$$

and $i, j=1, \ldots, 4$ run over the Higgs field components?. The matrix $A$ contains the usual combinatorical factors. The bright and dark circles are auxiliary vertices which are determined by the equation system. As the dot one can attach a matrix to each of them:

$$
\Gamma_{\text {bright }}^{i j}=\left(-i 2 g_{t}^{2} \zeta\right) \cdot K_{i j} \quad \Gamma_{\text {dark }}^{i j}=\left(-i 2 g_{t}^{2} \zeta\right) \cdot Q_{i j} .
$$

The full vertex

$$
\Gamma_{\text {full }}^{i j}=\left(-i 2 g_{t}^{2} \zeta\right) \cdot \Gamma_{i j}
$$

contains $\mathrm{s}^{-}$and $\mathrm{t}$-channel bubble sums and is therefore the sum of the bright and the dark vertex minus the double counted dot (fig. 13)].

Each loop integral $I$ in fig. 12 is calculated without combinatorical factors in the following way:

$$
I=\int \frac{d^{4} k}{(2 \pi)^{4}}\left(-i 2 g_{t}^{2} \zeta\right)^{2}\left(\zeta^{-1} \frac{i}{k^{2}}\right)\left(\zeta^{-1} \frac{i}{(q-k)^{2}}\right) .
$$

\footnotetext{
${ }^{6}$ E.g. $\Gamma_{\mathrm{SM}}^{12}$ is the four-boson-vertex of the Standard Model with two Higgs bosons $H$ and two Goldstone bosons $G^{0}$.

${ }^{7}$ The $\mathrm{t}$-channel bubble sum of the bright vertex inserted in the second diagram of fig. 10 provides graphs, which can be interpreted as boson self-energy corrections for the one-loop diagram. One could argue that this leads to a double counting of diagrams. But since the quadratic divergences of the whole diagram and the boson self-energy subdiagram have different origins there is not any double counting of quadratic divergences because we extract different terms.
} 

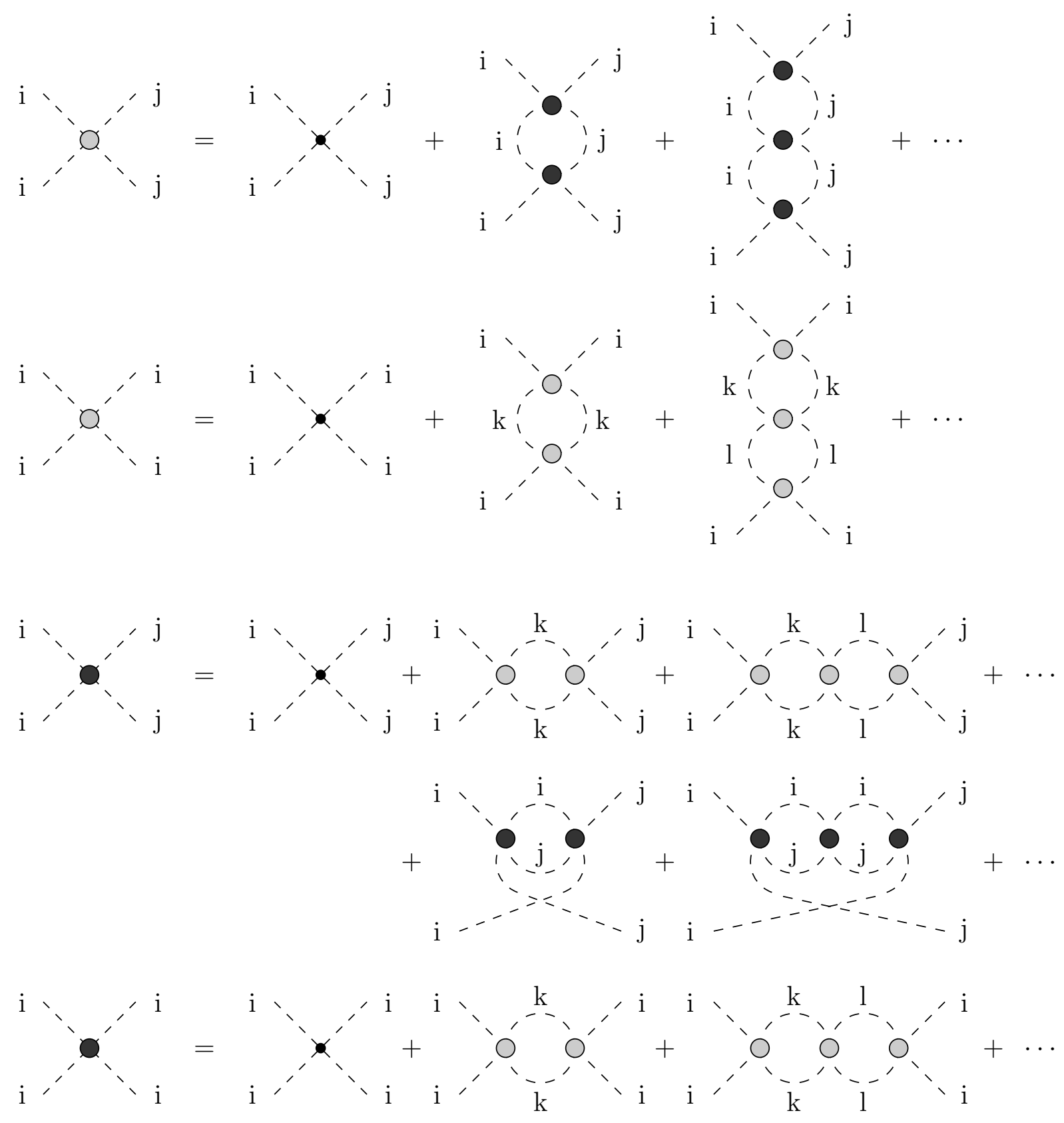

Figure 12: Equation system $\left(i, j, k, \ldots \in\left\{H, G^{0}, G^{1}, G^{2}\right\}\right.$ and $\left.i \neq j\right)$.

The leading divergence is extracted for large loop momentum $k$. Thus all $\zeta$ 's in the vertices and the propagators depend on the largest momentum $k$ and cancel therefore in this limit. 


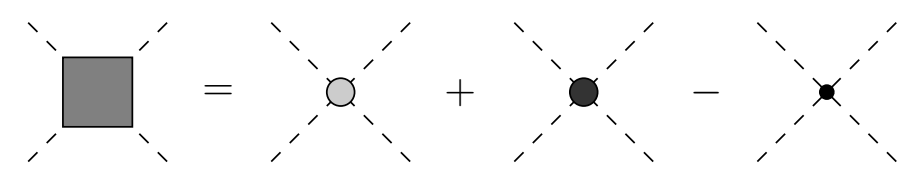

Figure 13: Equation for the full vertex.

Hence we get:

$$
I=i \frac{4 g_{t}^{4}}{(4 \pi)^{2}} \ln \frac{M^{2}}{p^{2}}=\left(-\frac{2}{N_{c}}\right)\left(-i 2 g_{t}^{2} \zeta\right)
$$

up to finite terms, where $p$ is the largest momentum in the diagram 8 . This again has the form of a boson vertex with an additional factor $\left(-\frac{2}{N_{c}}\right)$. In this way we can calculate a complicated diagram step by step.

Including all combinatorical factors the equation system of fig. 12 reads now $(i, j$ fixed, $i \neq j)$ :

$$
\begin{aligned}
K_{i i}=Q_{i i}=A_{i i} & -\frac{2}{N_{c}}\left(K^{2}\right)_{i i}+\frac{2}{N_{c}^{2}}\left(K^{3}\right)_{i i}-\frac{2}{N_{c}^{3}}\left(K^{4}\right)_{i i}+-\ldots \\
K_{i j}=A_{i j} & -\frac{4}{N_{c}}\left(Q_{i j}\right)^{2}+\frac{8}{N_{c}^{2}}\left(Q_{i j}\right)^{3}-\frac{16}{N_{c}^{3}}\left(Q_{i j}\right)^{4}+-\ldots \\
Q_{i j}=A_{i j} & -\frac{1}{N_{c}}\left(K^{2}\right)_{i j}+\frac{1}{N_{c}^{2}}\left(K^{3}\right)_{i j}-\frac{1}{N_{c}^{3}}\left(K^{4}\right)_{i j}+-\ldots \\
& -\frac{2}{N_{c}}\left(Q_{i j}\right)^{2}+\frac{4}{N_{c}^{2}}\left(Q_{i j}\right)^{3}-\frac{8}{N_{c}^{3}}\left(Q_{i j}\right)^{4}+-\ldots
\end{aligned}
$$

and the full vertex of fig. 13 has the form:

$$
\Gamma=\frac{1}{2}(K-A)+(Q-A)+A
$$

where we have to put in the solutions of the equation system?

8 This $p$ need not to be $q$ but can also come from the infrared behaviour of a $\zeta$.

${ }^{9}$ In line 1 and 3 of the system (25) the factor 2 of the expansion parameter $-\frac{2}{N_{c}}$ is missing since each boson-loop gets a combinatorical factor $1 / 2$. In addition line 1 and 2 have an overall factor 2 . This considers the fact that a reinsertion of a bubble sum destroys a combinatorical factor $1 / 2$ in the underlying bubble sum. That factor 2 must be removed in the vertex equation (26) since there is no further insertion in a bubble sum. 
The equations (25) are very complicated. It is reasonable to make an Ansatz for the matrices $K$ and $Q$. We use the assumption that the solution is invariant under the exchange of two Higgs field components. In that case $K$ and $Q$ have the following simple form:

$$
K=\left(\begin{array}{llll}
x & y & y & y \\
y & x & y & y \\
y & y & x & y \\
y & y & y & x
\end{array}\right) \quad, \quad Q=\left(\begin{array}{llll}
u & v & v & v \\
v & u & v & v \\
v & v & u & v \\
v & v & v & u
\end{array}\right) .
$$

The equations (25) and (26) can be simplified to:

$$
\begin{aligned}
3 x & =3 u=3+2 N_{c}-2 N_{c} \cdot \frac{1+\frac{x}{N_{c}}+2 \frac{y}{N_{c}}}{\left(1+\frac{x}{N_{c}}\right)^{2}+2\left(1+\frac{x}{N_{c}}\right) \frac{y}{N_{c}}-3\left(\frac{y}{N_{c}}\right)^{2}} \\
y & =1-\frac{\frac{4}{N_{c}} v^{2}}{1+\frac{2}{N_{c}} v} \\
2 v & =1-y+\frac{2 y}{\left(1+\frac{x}{N_{c}}\right)^{2}+2\left(1+\frac{x}{N_{c}}\right) \frac{y}{N_{c}}-3\left(\frac{y}{N_{c}}\right)^{2}}
\end{aligned}
$$

and

$$
\Gamma_{i i}=3\left(\frac{x}{2}-\frac{1}{2}\right) \quad \Gamma_{i j}=\frac{y}{2}+v-\frac{1}{2} .
$$

The best way to solve this system is to subtract the third from the first equation in (28). One gets:

$$
(x-y)^{2}-(x-y) \frac{2}{3}\left(v-y+1-\frac{N_{c}}{2}\right)-\frac{2}{3} N_{c}(v-y+1)=0
$$

and

$$
(x-y)^{2}-(x-y) \frac{\frac{4}{N_{c}} v^{2}-\frac{N_{c}}{3}}{1+\frac{2}{N_{c}} v}-N_{c} \frac{\frac{4}{N_{c}} v^{2}+\frac{2}{3} v}{1+\frac{2}{N_{c}} v}=0
$$

after replacing of $y$ by the second equation in (28). This equation has two types of solutions:

$$
\begin{aligned}
& x-y=2 v \\
& x-y=-\frac{\frac{N_{c}}{3}+2 v}{1+\frac{2}{N_{c}} v} .
\end{aligned}
$$

The solution (32) is significant for the full vertex $\Gamma$. It gives $\Gamma_{i i}=3 \cdot \Gamma_{i j}$ so that

$$
\Gamma=\frac{x-1}{2} A,
$$


i. e. $\Gamma$ is proportional to the tree level vertex $\Gamma_{S M}$ (see eq. (20)), which guarantees the $\mathrm{O}(4)$-symmetry 10 of the full vertex $\Gamma$. The solution (33) does not work in the same way and breaks the $\mathrm{O}(4)$-symmetry explicitly, which we will not further consider.

The equation system (28) together with eq. (32) leads to:

$$
6 x^{3}-2\left(N_{c}+6\right) x^{2}-\left(N_{c}^{2}+4 N_{c}-6\right) x+3 N_{c}^{2}+6 N_{c}=0 .
$$

For the physical relevant case $N_{c}=3$ we have the three solutions:

$$
x_{1}=3 \quad x_{2}=\sqrt{\frac{5}{2}} \quad x_{3}=-\sqrt{\frac{5}{2}} .
$$

After insertion of the first solution in eq. (34) one gets:

$$
\Gamma=A
$$

which is the desired result that the full vertex is just the fermion-loop induced vertex. This means that the graphs in fig. 10 and fig. 9 give the same result for the leading divergences. The quadratic divergences cancel therefore automatically as in the preliminary approximation. The other solutions in eq. (36) are energetically suppressed since they cannot lower the top mass many orders of magnitude.

It is a quite amazing result after the penetration across a technical jungle and one has to ask for a simple understanding. In such a case one usually presumes a hidden symmetry responsible for the cancellation. In fact it is more complicated than expected because only one solution may have this symmetry while the other ones provide quadratic divergences. Moreover the $\mathrm{x}$-value of this solution and therefore the boson loop diagram of fig. 10 grows proportional to $N_{c}$ which contradicts a simple counting of powers of $N_{c}$. Thus the solution is non-perturbative in $1 / N_{c}$. A cancellation order by order is therefore excluded and the counting of degrees of freedom is non-trivial. The following generalization of the model sheds some light on the subject.

\section{General cancellation condition}

One can ask whether a cancellation occurs for more general gauge group combinations. For a general framework one can also generalize the weak gauge group $S U(2)_{L}$ so that we

\footnotetext{
${ }^{10}$ The appearance of the $\mathrm{O}(4)$-symmetry in the solutions also confirms the correctness of the SchwingerDyson-system in fig. 12.
} 
get a general Standard Model:

$$
S U\left(N_{c}\right)_{c} \times S U\left(N_{L}\right)_{L} \times U(1)_{Y}
$$

This means that $\psi_{L}$ in the top condensation Lagrangian (2) has $N_{L}$ components and therefore $N_{L}-1$ left-handed b-quarks:

$$
\psi_{L}=\left(\begin{array}{c}
t_{L} \\
b_{L, 1} \\
\vdots \\
b_{L, N_{L}-1}
\end{array}\right)
$$

The $S U\left(N_{L}\right)_{L}$ is broken at a high scale in such a way that each left-handed b-quark can only couple to the $t_{L}$ at the electroweak scale, so that we have $2 N_{L}-1$ massless vectorbosons. In other words: we have $N_{L}-1 S U(2)_{L}$-subgroups with one Z-boson and $2 N_{L}-2$ $W$-type vector-bosons. They acquire a mass by the top condensation mechanism, which contains now $2 N_{L}$ Higgs field components $H, G^{0}, G_{1}^{+}, G_{1}^{-}, \ldots, G_{N_{L}-1}^{+}, G_{N_{L}-1}^{-}$, which are the boundstates $\bar{t} t, \bar{t} \gamma^{5} t, \bar{b}_{L, 1} t_{R}, \bar{t}_{R} b_{L, 1}, \ldots, \bar{b}_{L, N_{L}-1} t_{R}, \bar{t}_{R} b_{L, N_{L}-1}$, so that $A, K$ and $Q$ are $2 N_{L} \times 2 N_{L}$-matrices:

$$
A=\left(\begin{array}{cccc}
3 & 1 & \cdots & 1 \\
1 & \ddots & \ddots & \vdots \\
\vdots & \ddots & \ddots & 1 \\
1 & \ldots & 1 & 3
\end{array}\right) \quad K=\left(\begin{array}{cccc}
x & y & \cdots & y \\
y & \ddots & \ddots & \vdots \\
\vdots & \ddots & \ddots & y \\
y & \ldots & y & x
\end{array}\right) \quad Q=\left(\begin{array}{cccc}
u & v & \cdots & v \\
v & \ddots & \ddots & \vdots \\
\vdots & \ddots & \ddots & v \\
v & \ldots & v & u
\end{array}\right)
$$

Hence the system (28) has the form:

$$
\begin{aligned}
3 x & =3 u=3+2 N_{c}-2 N_{c} \cdot \frac{1+\frac{x}{N_{c}}+\left(2 N_{L}-2\right) \frac{y}{N_{c}}}{\left(1+\frac{x}{N_{c}}\right)^{2}+\left(2 N_{L}-2\right)\left(1+\frac{x}{N_{c}}\right) \frac{y}{N_{c}}-\left(2 N_{L}-1\right)\left(\frac{y}{N_{c}}\right)^{2}} \\
y & =1-\frac{\frac{4}{N_{c}} v^{2}}{1+\frac{2}{N_{c}} v} \\
2 v & =1-y+\frac{2 y}{\left(1+\frac{x}{N_{c}}\right)^{2}+\left(2 N_{L}-2\right)\left(1+\frac{x}{N_{c}}\right) \frac{y}{N_{c}}-\left(2 N_{L}-1\right)\left(\frac{y}{N_{c}}\right)^{2}} .
\end{aligned}
$$

The general equation for eq. (35) is

$$
2\left(N_{L}+1\right) x^{3}-2\left(N_{c}+2 N_{L}+2\right) x^{2}+\left(2-2 N_{L}\left(N_{c}-1\right)-N_{c}^{2}\right) x+2 N_{c}\left(N_{L}+1\right)+3 N_{c}^{2}=0
$$

and the general cancellation condition reads

$$
\frac{x-1}{2}=\frac{N_{c}}{N_{L}+1} .
$$


After the elimination of $x$ in eq. (42) and (43) we find the simple condition:

$$
N_{c}=\left(N_{L}+1\right) \frac{N_{L}-1}{3-N_{L}} .
$$

One easily sees that the only reasonable and possible combination is:

$$
N_{c}=3 \quad, \quad N_{L}=2 .
$$

We recognize that the Standard Model is the only possible choice of gauge groups leading to a cancellation of quadratic divergences in its top condensation extension.

In eq. (44) a special feature of our solution is visible: For $N_{L}=3 N_{c}$ will be infinite. One would expect that in this case the lowest order $1 / N_{c}$ yields the exact results. But there is no cancellation of quadratic divergences in lowest order since no boson-loop graphs appear. The reason for the cancellation is that the above solution is non-perturbative in $1 / N_{c}$, i. e. even, if $N_{c}$ goes to infinity, higher order contributions are necessary to include.

From eq. (44) we further see that the reason for the cancellation is more complicated than counting degrees of freedom as in supersymmetry. The condition (44) rather reminds one of the anomaly cancellation. One can try to find a general anomaly condition for our model. Since $S U\left(N_{L}\right)_{L}$ is broken and each $b_{i, L}$ couples only to the $t_{L}$ one can simply associate the top with the charge $2 / 3$ and all $b$ 's with the charge $-1 / 3$. In the same way we have one chargeless tau-neutrino and $N_{L}-1$ taus with charge -1 . Thus the anomaly cancellation reads:

$$
N_{c}\left[\frac{2}{3}-\frac{1}{3} \cdot\left(N_{L}-1\right)\right]+\left[0-1 \cdot\left(N_{L}-1\right)\right]=0
$$

or

$$
N_{c}=3 \cdot \frac{N_{L}-1}{3-N_{L}} .
$$

Although there is no obvious connection, the conditions (44) and (47) have a surprising similarity.

\section{Vector-boson contributions}

In fig. 9 we have neglected the vector-boson contributions. In the Standard Model in lowest order of the gauge couplings one has to add the $Z^{0}$ - and $W^{ \pm}$-induced Higgs self-energies in fig. 1, where in lowest order top condensation the four-boson-vertex again must be 


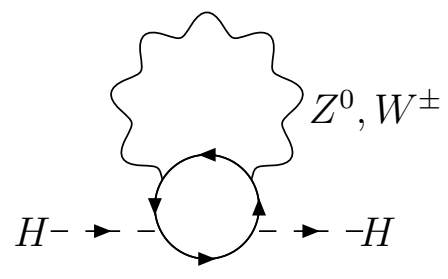

Figure 14: A possible vector-boson contribution.

replaced by a fermion-loop (see fig. 14). These diagrams seem to destroy our cancellation mechanism since they provide additional quadratic divergences with a negative sign. But these diagrams are suppressed at least by a factor $M_{W}^{2} / m_{t}^{2}$ for each vector-boson line. One can therefore use perturbation theory to extract the vector boson contributions. On the contrary both graphs in fig. 9 are of the same order in top condensation and the vectorboson graph in fig. 14 can be regarded as a quantum correction to the first diagram in fig. 9. Thus a quantum correction to the second diagram in fig. 9 is of the same order as the graph in fig. 14 but has the opposite sign. We get therefore vector contributions with both signs. Most of these corrections are self-energies or vertex corrections, which enter the top mass and the top Yukawa coupling. Hence they drop out for the quadratic divergences.

Although one cannot prove the cancellation of the quadratic divergences of the vectorboson graphs diagrammatically to all orders, the above consideration shows that these diagrams do not directly provide quadratic divergences as in the Standard Model. They can only appear as quantum corrections. But even if the cancellation mechanism is broken by radiative corrections, a smaller hierarchy structure remains, i. e. nevertheless the top condensation scale could be much larger than $1 \mathrm{TeV}$.

\section{Conclusion}

We have discussed the hierarchy problem and the corresponding quadratic divergences in top condensation models. As we know from supersymmetry a cancellation of these quadratic divergences needs certain relations for the couplings and the degrees of freedom between the fermions and the bosons. In supersymmetry this is guaranteed by symmetry. 
Here compositeness leads to a relation between the top Yukawa-coupling and the Higgs self-coupling in top condensation. In analogy to the Veltman condition, which has a similar structure, a cancellation of quadratic divergences should appear, at least partly. Nambu called such a scenario "Quasi-Supersymmetry", because of the partly cancellation of the top and the Higgs loop diagrams, but here we could not identify a symmetry as the origin of the cancellation. The exact cancellation is not obvious and needs a precise consideration of the contributing diagrams. The $1 / N_{c}$-expansion does not respect the Veltman-like cancellation since fermion and boson-loop diagrams are of different order $1 / N_{c}$. Moreover the boson-loop diagrams are hidden in the full Schwinger-Dyson-equation and must be extracted by reshuffling of the Feynman diagrams. In a preliminary approximation we found that the fermion and boson-loop graphs in fig. 9 are free of quadratic divergences. This cancellation does however not lead to a Veltman-like relation between the top and the Higgs mass.

To demonstrate the cancellation to all orders we have shown that the full four-boson-vertex is just the fermion-loop so that the leading quadratic divergences of fig. 10 cancel in the same way as in fig. 9. The full vertex is a solution of a complicated system of SchwingerDyson-equations. It contains indeed a solution where a miraculous cancellation happens. The fact that it only works for $N_{c}=3$ and $N_{L}=2$ means that a possible Grand Unified gauge group at the Grand Unification scale prefers the Standard Model gauge group, since the masses of the Standard Model can be many orders of magnitude smaller than in other breaking schemes. If a symmetry could be found being responsible for the cancellation mechanism, it would be non-trivial and would have a complicated structure since it works only for a special solution of the Schwinger-Dyson-equations. The cancellation rather seems to come from a counting of fermionic and bosonic degrees of freedom depending on the color, flavor and other statistical factors. Although there is no better understanding for this mechanism, we made some consistency checks for the four-boson-vertex and the Schwinger-Dyson-equations and the cancellation seems to work.

In this paper we have concentrated our analysis on top condensation models, which have the four-fermion-interaction (2) in the low energy limit, especially the topcolor-model by C. T. Hill. Other models use a different color structure, where color is summed between equal quark flavors. The quadratic divergences of such models can in principle be calculated. It requires an additional analysis of the color structure in each Feynman diagram, but a similar cancellation of quadratic divergences is not excluded.

This cancellation mechanism is further not restricted on top condensation. It can probably be applied to other composite Higgs models. A cancellation of quadratic divergences in 
the low energy limit of some gauge theories in the technicolor scenario would admit a technicolor scale above the TeV-range. But especially the naturalness of top condensation would show that a dynamical origin of the electroweak symmetry breaking enables a simple explanation of the mass hierarchies.

Acknowledgement: I would like to thank M. Beneke, M. Hutter, M. Misiak and B. Stech for useful discussions and C. T. Hill and M. Lindner for useful comments on the draft version of this paper. 


\section{References}

[1] R. Decker and J. Pestieau, UCL-ITP-79-19 (1979);

M. Veltman, Acta Phys. Pol. B12 (1981) 437.

[2] H. P. Nilles, Phys. Rep. 110 (1984) 1;

H. E. Haber and G. L. Kane, Phys. Rep. 117 (1985) 75;

M. F. Sohnius, Phys. Rep. 128 (1985) 39.

[3] M. S. Al-sarhi, I. Jack and D. R. T. Jones, Z. Phys. C55 (1990) 283;

P. Osland and T. T. Wu, Phys. Lett. B291 (1992) 315 and

Z. Phys. C55 (1992) 569, 585, 593;

K. Just and L. S. The, Z. Naturforsch. A42 (1987) 241;

E. Ma, UCRHEP-T100 (1992);

I. H. Lee and S. D. Drell, SLAC-PUB-5423 (1991);

A. Blumhofer and B. Stech, Phys. Lett. B293 (1992) 137.

[4] M. Ruiz-Altaba, B. Gonzales and M. Vargas, CERN-TH.5558/89 (1989);

M. Capdequi Peyranere et al., Phys. Lett. B260 (1991) 138.

[5] I. Jack and D. R. T. Jones, Phys. Lett. B234 (1990) 321;

I. Jack and D. R. T. Jones, Nucl. Phys. B342 (1990) 127;

M. S. Al-sarhi, I. Jack and D. R. T. Jones, Nucl. Phys. B345 (1990) 431.

[6] Y. Nambu, in New Theories in Physics, Proc. XI Int. Symposium on Elementary Particle Physics, Warsaw (World Scientific, Singapore, 1989), EFI 85-86 (1985) and EFI 88-39 (1988).

[7] V. Miransky, M. Tanabashi and Y. Yamawaki, Phys. Lett. B221 (1989) 177 and Mod. Phys. Lett. A4 (1989) 1043;

W. Marciano, Phys. Rev. Lett. 62 (1989) 2793;

W. A. Bardeen, C. T. Hill and M. Lindner, Phys. Rev. D41 (1990) 1647.

[8] A. Blumhofer, Phys. Lett. B320 (1994) 352.

[9] A. Hasenfratz et al. , Nucl. Phys. B365 (1991) 79;

U. Ellwanger and L. Vergara, Nucl. Phys. B398 (1993) 52;

M. Lindner, Intern. J. Mod. Phys. A8 (1993) 2167. 
[10] S. King and S. Mannan, Phys. Lett. B241 (1990) 249;

C. T. Hill, Phys. Lett. B266 (1991) 419;

R. Bönisch, Phys. Lett. B268 (1991) 394;

M. Lindner and D. Ross, Nucl. Phys. B370 (1992) 30. 